\title{
The picture of relativistic jet from Fermi-LAT and multi-band observations of blazar 3C 279
}

\author{
Masaaki Hayashida ${ }^{1}$ and Greg Madejski ${ }^{1}$ for the Fermi-LAT \\ collaboration and members of 3C 279 multi-band campaign \\ ${ }^{1}$ Kavli Institute for Particle Astrophysics and Cosmology, SLAC National Accelerator \\ Laboratory, Stanford University, CA, 94025, USA \\ email: mahaya@slac.stanford.edu
}

\begin{abstract}
Strong and variable radiation detected over all accessible energy bands in blazar arises from a relativistic, Doppler-boosted jet pointing close to our line of sight. Flat Spectrum Radio Quasar 3C 279 was one of the brightest $\gamma$-ray blazars in the sky at the time of the discovery with EGRET. Since the successful launch of the Fermi Gamma-ray Space telescope in 2008 , we have organized extensive multi-band observational campaign of 3C 279 from radio to $\gamma$-ray bands, also including optical polarimetric observations. The uninterrupted monitoring in the $\gamma$-ray band by Fermi-LAT together with the multi-band data provide us with new insights of the relativistic jet of blazar. Here, we present the results of the first-year multi-band campaign of 3C 279 including the discovery of a $\gamma$-ray flare event associated with a dramatic change of the optical polarization - as well as a discovery of an "orphan" X-ray flare, unassociated with prominent outbursts in other bands.
\end{abstract}

Keywords. galaxies: jets - quasars: individual (3C 279) - gamma rays: observations - X-rays: galaxies - polarization

\section{Blazar 3C 279}

The flat spectrum radio quasar 3C 279 was the first bright $\gamma$-ray blazar reported by the EGRET instrument aboard the Compton Gamma-Ray Observatory to show strong and rapidly variable $\gamma$-ray emission (Hartman et al. 1992, Kniffen et al. 1994, Wehrle et al. 1998); recently, it also has been detected at photon energies above $100 \mathrm{GeV}$ by the MAGIC ground-based Cherenkov telescope (Albert et al. 2008). This blazar, at the redshift $z=0.536$, harbors a black hole with mass $M \simeq(3-8) \times 10^{8} M_{\odot}$ (Woo \& Urry 2002, Nilsson et al. 2009); for specificity, we adopt $6 \times 10^{8} M_{\odot}$. It shows superluminal expansion best described as the jet material propagating with the bulk Lorentz factor $\Gamma_{\text {jet }}=16 \pm 3$ at a small angle $\left(\theta \sim 2^{\circ}\right)$ to our line of sight (Jorstad et al. 2005). In this paper, we report the results of the first-year Fermi-LAT and multi-band campaign of 3C 279 including optical polarimetric observations.

\section{Multi-band observational campaign with Fermi-LAT}

The successful launch and deployment of the Fermi Gamma-ray Space Telescope inaugurated a new capability to monitor the $\gamma$-ray sky. The main instrument on-board Fermi, the Large Area Telescope (LAT: Atwood et al. 2009) features a wide (2.4 steradian) field of view and rocks by $35-50^{\circ}$ north and south of the orbital plane every second orbit, it observes the entire sky every $\sim 3$ hours, allowing an essentially uninterrupted flux history of $3 \mathrm{C} 279$ in the $\gamma$-ray band. The observations of the source were conducted in other 


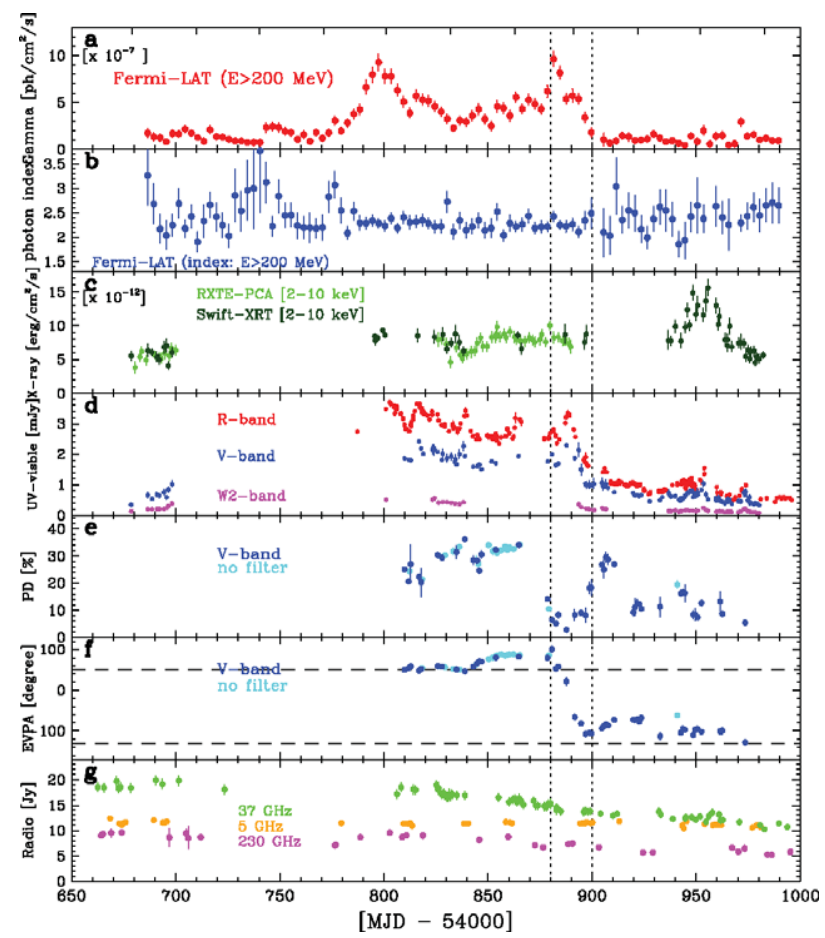

Figure 1. History of fluxes in $\gamma$-ray above $200 \mathrm{MeV}$ (a), X-ray of 2-10 keV (c), optical-UV (d), radio (g) bands as well as $\gamma$-ray photon index (b), optical polarization degree (e) and angle (f) of 3C 279 between July 2008 and June 2009 (54650 and 55000 MJD). The two dashed vertical lines correspond to 54880 and 54900 MJD, respectively. The full version of the light curves of the campaign including some additional bands can be seen in Abdo et al. (2010).

bands as well, and the best coverage was obtained after the start of the routine scientific operation of the Fermi-LAT (on August 4th 2008, 54682 MJD). In Figure 1 we plot the flux history in the $\gamma$-ray band by Fermi-LAT, as well as in the X-ray band measured by RXTE-PCA and Swift-XRT, optical, infrared and radio bands by the GASP-WEBT observatories $\dagger$ and Swift-UVOT. Polarization information in the optical band are also provided by Kanata (V-band:Watanabe et al. 2005) and KVA (no filter) telescopes.

\section{Gamma-ray flare with a change of optical polarization}

Towards the end of the high state there is a sharp $\gamma$-ray flare at 54880 MJD. This sharp $\gamma$-ray flare coincides with a significant drop of the level of optical polarization (polarization degree: PD), from $\sim 30 \%$ down to a few $\%$, lasting for $\Delta t \sim 20$ days. Subsequently, both $\gamma$-ray and optical fluxes gradually decrease together and reach the quiescent level, followed by a temporary recovery of the high degree of polarization. This event is associated with a dramatic change of the electric vector position angle (EVPA) of the polarization, in contrast to being relatively constant before the event at $\sim 50^{\circ}$ (parallel to the jet direction observed by Very Long Baseline Interferometry observations in radio bands; see, e.g., Jorstad et al. 2005). Since the EVPA has $\pm 180^{\circ} \times n$ ambiguity, we selected values on the assumption of a smooth change of the EVPA, such that it would follow the overall trend. The polarization angle increases slightly at 54880 MJD - coincident with the $\gamma$-ray flare - then decreases by $208^{\circ}$ with an average rate of $\sim 12^{\circ}$ per day, and returns

$\dagger$ See details of the program in http://www.to.astro.it/blazars/webt/gasp/homepage.html 


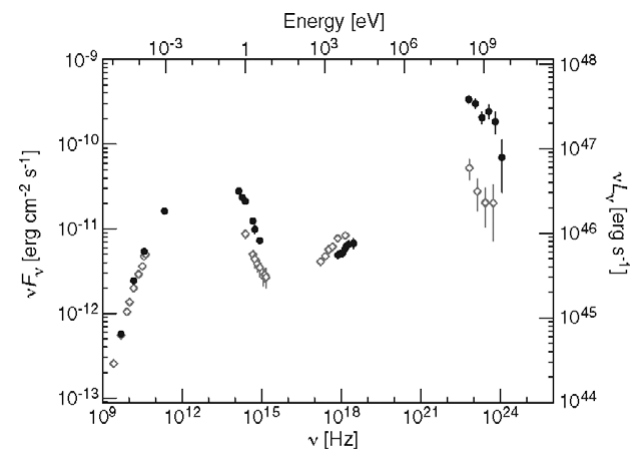

Figure 2. Energy spectrum from radio to $\gamma$-ray band of 3C 279 at two different epochs. The filled circle points were taken between 54880 and 54885 MJD, corresponding to the first five days of the sharp $\gamma$-ray flare accompanying the dramatic polarization change event while the open diamond points were taken between 54950 and 54960 MJD, around the peak of the isolated X-ray flare. The original version is taken from Abdo et al. (2010).

to a level nearly exactly $180^{\circ}$ from the original level, resembling closely the behavior of optical polarization measured in BL Lacertae (Marscher et al. 2008), but at a 4 times slower rate. This clearly indicates that the sharp $\gamma$-ray flare is unambiguously correlated with the dramatic change of optical polarization due to a single, coherent event, rather than a superposition of multiple shorter duration events.

The gradual rotation of the polarization angle is unlikely to originate in an axially symmetric jet since any compression of the jet plasma by, e.g., a perpendicular shock moving along the jet and viewed at a small but constant angle to the jet axis would change the degree of polarization, but would not result in a gradual change of EVPA. Instead, it can reflect a non-axisymmetric magnetic field distribution (as in, e.g., Konigl $\&$ Choudhuri 1985). The first possibility may be due to propagation of an emission knot following a helical path in a magnetically dominated jet as was recently investigated in the context of the optical polarization event seen in BL Lacertae (Marscher et al. 2008) or may involve the "global" bending of a jet. Since the magnetic field in the emission region is anisotropic - presumably concentrated in the plane of a shock / disturbance propagating along the jet - the degree and angle of observed polarization then depends on the instantaneous angle $\theta$ of the direction of motion of the radiating material to the line of sight. The maximum rotation rate of the polarization angle would correspond to the minimum of $\theta$. Similar geometry - albeit on larger scales - has been observed in another blazar, PKS 1510-089 (Homan et al. 2002). Nonetheless, in both scenarios, the coherent polarization event is produced by a density pattern co-moving along the jet, and therefore, it is possible to estimate the distance traveled by the emitting material during the flare $\Delta r_{\text {event }}$; this in turn allows us to constrain the distance of the dissipation region from the black hole $r_{\text {event }} \geqslant \Delta r_{\text {event }} \sim 10^{19}\left(\Delta t_{\text {event }} / 20\right.$ days $)\left(\Gamma_{\text {jet }} / 15\right)^{2} \mathrm{~cm}$, which is $\sim 5$ orders of magnitude larger than the gravitational radius of the black hole in $3 \mathrm{C} 279$. The constraints on the distance of the dissipation region can be relaxed under "flow-through" scenarios - where the emission patterns may move much slower than the bulk speed of the jet or not propagate at all: one such example is the model involving swings ("wobbling") of the jet associated with jet instabilities such that its boundary moves relative to our line of sight. In this case the time scale for the observed variation is the time scale for the jet motion. Consequently, the emission region easily can be much closer (by a factor $\Gamma_{\text {jet }}^{2}$ ) to the black hole than in the "helical" or "bent jet" scenarios, since the natural radial scale for $\Delta t_{\text {event }} \sim 20$ days is $r_{\text {event }} \sim c \Delta t_{\text {event }} \sim 500-1000$ gravitational radii (see, e.g., Lyutikov et al. 2003). 
This leaves us with three viable possibilities. Both the scenario involving a knot propagating along the helical magnetic field lines and the "flow-through" scenario above imply that the rotation of the polarization angle should be preferentially following the same direction, since in those two models the twist presumably originates in the inner accretion disk. In our case, we observe the rotation of the polarization angle that is opposite in direction than that measured previously (Larionov et al. 2008), leaving us with the "bent jet" model combined with a small swing of the jet as the most compelling.

\section{Isolated X-ray flare}

Concurrent X-ray observations indicate relatively steady flux during the $\gamma$-ray flare with the optical polarization change, but reveal a significant, symmetrical flare at 54950 MJD with duration of $\sim 20$ days, similar to the duration of the $\gamma$-ray flare. It suggests the X-ray photons are produced at a comparable distance from the black hole as the optical $/ \gamma$-ray photons. Importantly, this X-ray flare is not accompanied by a prominent optical or $\gamma$-ray flare. The X-ray spectrum during the isolated flare remains much harder than the optical spectrum (see Figure 2), and therefore cannot be attributed to a temporary extension of the high-energy tail of the synchrotron emission, but instead, may be generated by inverse-Compton scattering of low-energy electrons. However, the similarity of profiles of the $\gamma$-ray and X-ray flares argues against the latter being just a delayed version of the former due to, e.g., particle cooling. Therefore, it must be produced independently by another mechanism involving primarily lower energy electrons.

More details of the campaign results and discussions are described in Abdo et al. (2010).

The Fermi-LAT Collaboration acknowledges support from a number of agencies and institutes for both development and the operation of the LAT as well as scientific data analysis. These include NASA and DOE in the United States, CEA/Irfu and IN2P3/CNRS in France, ASI and INFN in Italy, MEXT, KEK, and JAXA in Japan, and the K. A. Wallenberg Foundation, the Swedish Research Council and the National Space Board in Sweden. Additional support from INAF in Italy and CNES in France for science analysis during the operations phase is also gratefully acknowledged.

\section{References}

Abdo, A. A., et al. Nature, 463, 919-923 (2010).

Albert, J., et al. Science, 320, 1752-1754 (2008).

Atwood, W. B., et al. Astrophys. J., 697, 1071-1102 (2009).

Dermer, C., Schlickheiser, R., \& Mastichiadis, A. Astron. Astrophys., 256, L27-L30 (1992).

Hartman, R. C., et al. Astrophys. J. (Lett.), 385, L1-L4 (1992).

Homan, D. C., et al. Astrophys. J., 580, 742-748 (2002).

Jorstad, S. G., et al. Astron. J., 130, 1418-1465 (2005).

Kniffen, D. A., et al. Astrophys. J., 411, 133-136 (1994).

Konigl, A. \& Choudhuri, A. R. Astrophys. J. 289, 188-192 (1985).

Larionov, V. M., et al. Astron. Astrophys. 492, 389-400 (2008).

Lyutikov, M., Pariev, V. I., \& Blandford, R. Astrophys. J. 597, 998-1009 (2003)

Marscher, A. P., et al. Nature, 452, 966-969 (2008).

Nilsson, K., et al. Astron. Astrophys., 505, 601-604 (2009)

Watanabe, M., et al. Pub. Astron. Soc. Pacif., 117, 870-884 (2005).

Wehrle, A. E., et al. Astrophys. J., 497, 178-187 (1998).

Woo, J.-H. \& Urry, C. M. Astrophys. J., 579, 530-544 (2002). 


\section{Discussion}

BEDNAREK: In the LAT spectrum during the flare consistent with the MAGIC spectrum, you model only the LAT spectrum?

HAYASHIDA: The exptrapolation of our LAT spectrum to higher energies goes to below the MAGIC data points. But they are not simultaneous data and the LAT spectrum is still 4-5 times lower than the historical maximum flux among the EGRET observations.

Romero: Changes in the polarization angle were observed long before Fermi launching. Actually, Ileana Andruchow has published in A\&A the very same model you have shown here. It would be nice, I think, if the Fermi team checked the relevant literature. Just a comment.

HAYASHIDA: Thank you for your pointing out. In fact, we have been aware of your work on optical polarization observations. However, our work focuses in the long term monitoring and the event of a gradual and large rotation (about $180^{\circ}$ ) in polarization degree, while that paper has results of short term (4 days) and discusses only microvariability. It is rather different from our work and we did not have room for listing all previous polarimetric studies. We appreciate your work and we would like to emphasize the important of polarimetric observations.

ANDRUCHOw: Did you perform any statistical tests in order to analyze the variability in an isolated curve?

HAYASHIDA: When we apply chi-squared tests, for example, fitting with a constant flux, probability is almost zero, as one can easily expect from light curves even by eyes. Then we also calculated the fractional variability index to quantify the variability. Although the data sampling rates are not the same along different bands, the gamma-ray band shows the largest value. 